\title{
Novel Nonstationarity Assessment Method for Hypersonic Flutter Flight Tests
}

\author{
Hua Zheng (D), Junhao Liu, and Shiqiang Duan \\ School of Power and Energy, Northwestern Polytechnical University, Shaanxi, Xian 710072, China \\ Correspondence should be addressed to Hua Zheng; isea_zh@mail.nwpu.edu.cn
}

Received 6 June 2018; Revised 29 September 2018; Accepted 10 October 2018; Published 25 October 2018

Academic Editor: Pawel Packo

Copyright (C) 2018 Hua Zheng et al. This is an open access article distributed under the Creative Commons Attribution License, which permits unrestricted use, distribution, and reproduction in any medium, provided the original work is properly cited.

\begin{abstract}
Hypersonic aircraft have been rapidly developed in recent years both theoretically and experimentally. Aerothermoelastic simulation is very challenging due to its inherent complexity, but physical tests are a workable approach. Flutter tests with variable speed are a popular alternative to hypersonic tests which provide nonstationary structural response data. This paper proposes a nonstationarity assessment method based on energy distribution in the time-frequency domain. The proposed method reveals the nonstationarity level corresponding to the appropriate modal identification algorithm or flutter boundary prediction (FBP) method. Several classic flutter criteria are utilized to build a hypersonic aircraft FBP framework. Numerical simulation and experimental applications demonstrate the effectiveness and feasibility of the proposed method, which facilitates accurate flutter predictions for the subcritical turbulence response during hypersonic flutter flight.
\end{abstract}

\section{Introduction}

Flutter quality is a significant issue in the development of hypersonic aircraft. Problematic aspects of hypersonic flight include extreme aerodynamic heating and complex hypersonic flow field effects [1-3]. Numerical simulation is an economic (and popular) research method, but the environmental conditions of physical flight experiments are rarely simulated. The causes of nonlinearity and complexity in hypersonic flows, such as real gas effects, boundary layershock wave interactions, changes in material properties due to aerodynamic heating, and nonlinear aeroelastic interactions between the flow field and structures $[4,5]$, as well as the monolithic coupling of aerodynamic thermal characteristics, aerodynamic force, elastic force, and inertia, are highly complex. The partitioned simulation method is currently the most often applied approach to hypersonic aircraft research, but it unfortunately requires that weak coupling characteristics be ignored per the limited capability of existing software programs.

Many hypersonic aerothermoelastic experimental studies have been conducted over the past several decades [6-9]. The flutter behavior of various wing and rudder models has been determined (e.g., airfoil shape, airfoil thickness, attack angle, leading-edge bluntness, and wing-body interference) [10-13]. Existing test and data processing methods are a workable foundation for further theoretical development hypersonic aircraft simulation techniques. "Flutter" is a phenomenon characterized by system instability due to structural modal coupling [14-16] as evidenced by the structural response of undamped oscillation. In a hypersonic flutter test, structural responses directly reflect the process of modal change and energy alternation; the responses provide useful indicators of various flutter trends. Structural response signal analysis can also help to further improve both simulation techniques as well as innovative structure designs. There is, to this effect, urgent demand for effective hypersonic flutter flight test data processing methods.

Flutter flight tests are applied to conventional aircraft by launching several flight stages with different airspeeds to approximate the aerodynamic force as a stationary excitation. Several algorithms for modal parameter identification or flutter boundary prediction (FBP) have been established based on the stationary stochastic process theory; they include fast Fourier transform (FFT) [17, 18], random decrement technique (RDT) [19], natural excitation technique combined 
TABLE 1: Prediction results of different criteria.

\begin{tabular}{lcc}
\hline $\begin{array}{l}\text { System stability } \\
\text { criterion }\end{array}$ & \multicolumn{2}{c}{ True Value $=4451$} \\
\hline $\begin{array}{l}\text { Lyapunov's } \\
\text { Criterion }\end{array}$ & & \\
M(1) & 4531 & $0.44 \%$ \\
$\mathrm{M}(2)$ & 4459 & $2.02 \%$ \\
$\mathrm{M}(3)$ & 4523 & $1.62 \%$ \\
$\mathrm{M}(4)$ & 4593 & $0.92 \%$ \\
Jury's & & \\
Criterion & & \\
$F^{+}(3)$ & 4525 & $0.57 \%$ \\
$F^{-}(1)$ & 4565 & $0.31 \%$ \\
$F^{-}(3)$ & 4547 & $0.09 \%$ \\
\hline
\end{tabular}

with the Eigen system realization algorithm (NExT-ERA) [20], time series analysis based on the auto-regressive (AR) model [21], stochastic subspace identification (SSI) methods [22], and Hilbert-Huang Transform method [23].

Hypersonic aircraft have extremely short acceleration periods and very high Mach numbers. Flutter tests, however, require an efficient, effective progression of variable airspeeds. The structural response signal from such tests are nonstationary, and the solutions must be distinguished per the stationary situations. When there is low nonstationarity, the algorithms discussed above may be still feasible; otherwise, new methods for nonstationary signal processing are necessary for accurate estimation. There is not yet a standardized definition of nonstationarity - or any standard method for assessing it-due to inherent deficiencies in the mathematical theory.

This paper proposes a novel assessment theory for nonstationarity accompanied by classic flutter boundary prediction methods. A data processing framework for structural response was designed accordingly, and numerical simulations and experiments validate the proposed method and framework.

\section{Nonstationarity Level Assessment}

The nonstationarity level of the target signal directly affects the signal processing method and accuracy. There are several important aspects of nonstationarity, as discussed below.

(a) Degree of Cyclostationarity [24]. Cyclostationarity is defined by the distance between the autocorrelation function of periodic stationary signals $r_{x x}(t, \tau)$ and the generalized stationary autocorrelation function $r_{x x}(\tau)$ :

$$
\text { DCS } \stackrel{\text { def }}{=} \frac{\min _{r_{x x}(\cdot)} \int_{-\infty}^{\infty}\left\langle\left|r_{x x}(t, \tau)-r_{x x}(\tau)\right|^{2}\right\rangle d \tau}{\int_{-\infty}^{\infty}\left|\left\langle r_{x x}(t, \tau)\right\rangle\right|^{2} d \tau}
$$

That is, it is the integral of the least square error between normalized $r_{x x}(t, \tau)$ and $r_{x x}(\tau)$ according to the delay time $\tau$. (b) Hilbert Time-Frequency Spectrum [25]. The Hilbert spectrum of the signal $H(\omega, t)$ is first analyzed on the timefrequency plane; then a marginal spectrum is obtained:

$$
n(\omega)=\frac{1}{T} \int_{0}^{T} H(\omega, t) d t
$$

The nonstationarity level is defined as follows:

$$
D_{s}(\omega)=\frac{1}{T} \int_{0}^{T}\left(1-\frac{H(\omega, t)}{n(\omega)}\right)^{2} d t
$$

where nonstationarity is defined as a function of frequency, because certain frequency components in the signal may be nonstationary while others remain stationary.

(c) Correlation Integral [26]. Consider a time series $x_{t}, t=$ $1, \cdots N$ with the following correlation integral:

$$
I(N, R)=\frac{2}{N(N-1)} \sum_{i=1}^{N} \sum_{j=i+1}^{N} H\left(R-\left\|x_{i}-x_{j}\right\|\right)
$$

where $\mathrm{N}$ is the total number of samples, $\|\cdot\| \in \mathrm{L}_{\infty}$ is the norm space, $\mathrm{R}$ is the reference distance, and $\mathrm{H}$ is the unit step function.

For the stationary signal $y_{t}, t=0,1, \cdots N$, its distance sequence $y_{s}^{\prime}=\left\|y_{i}-y_{j}\right\|, s=0,1, \cdots, N(N-1) / 2$ is the normal distribution. The mathematical expectation of $y_{s}^{\prime}$ is $\mu=\mathrm{E}\left(y_{s}^{\prime}\right)$ and the standard deviation is $\sigma$. The nonstationarity of the assessed signal $x_{t}$ with the reference distance $R=\mu+2 \sigma$ is defined as follows:

$$
D=I_{y}(N, R)-I_{x}(N, R)
$$

The first of the above definitions relates to periodic nonstationary signals without generality; the second is computationally complex and does not have clear boundaries for stationary signals; the third applies only to specific engineering applications, which is hardly generalizable to the analysis of normal nonstationary signals.

For any signal $x(t)$, the time-frequency distribution based on the Hermite window function is

$$
S_{x, K}(t, f)=\frac{1}{K} \sum_{k=1}^{K}\left|\int x(s) h_{k}(s-t) e^{-i 2 \pi f s} d s\right|^{2}
$$

where $h_{k}(t)$ is the $k$ order Hermite function, defined as

$$
h_{k}(t)=\frac{H_{k}(t) \exp \left(-t^{2} / 2\right)}{\sqrt{2^{k} k ! \sqrt{\pi}}}
$$

Per the definition of "stationary", the first moment and secondary moment are independent of time:

$$
\begin{aligned}
E[x(t)] & =C \\
E[x(t+\tau) x(t)] & =g(\tau)
\end{aligned}
$$

where $\tau$ is the time delay and $E[\bullet]$ is the mathematical expectation. 


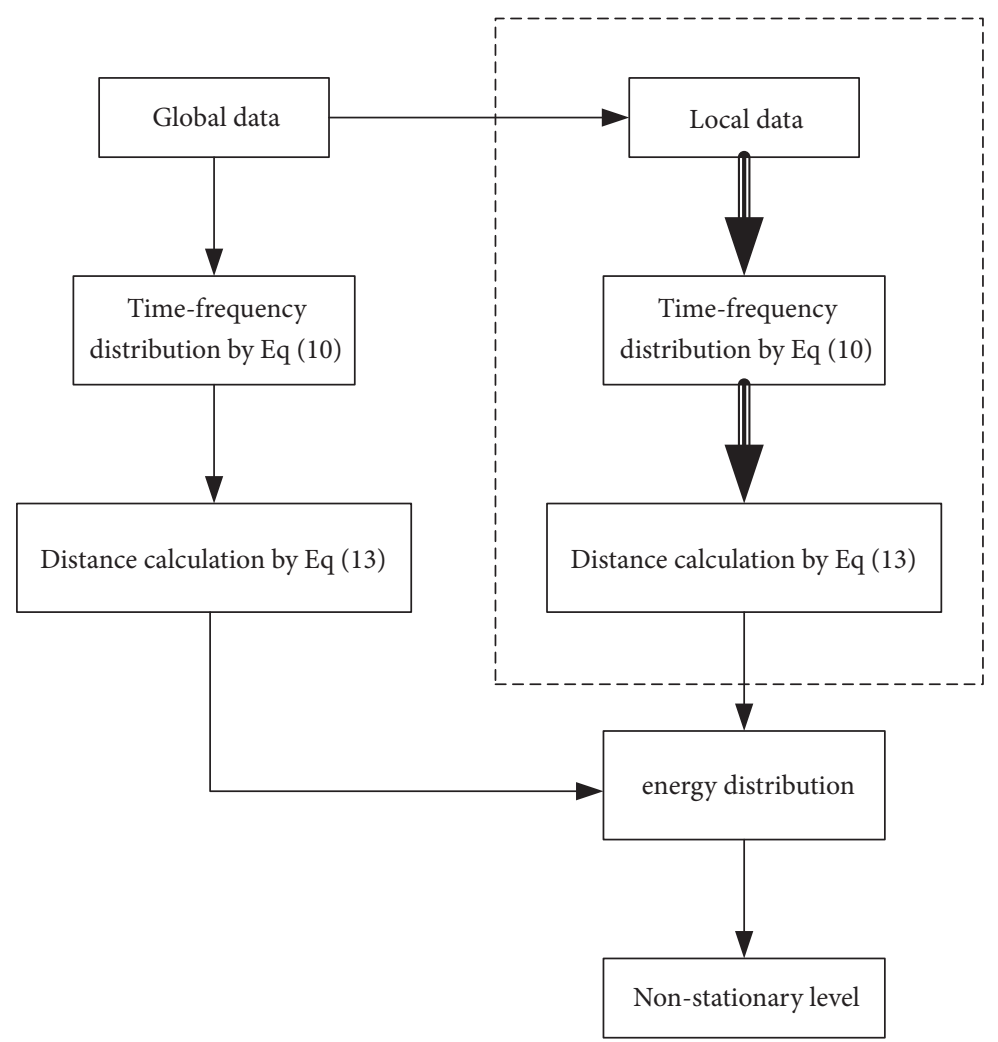

FIGURE 1: Flow chart: nonstationarity level assessment.

The local time-frequency distribution $S_{x, K}\left(t_{n}, f\right)$ must be equal to the mean of the global distribution at any time point. Their relationship is

$$
\left\langle S_{x}\left(t_{n}, f\right)\right\rangle=\frac{1}{N} \sum_{n=1}^{N} S_{x}\left(t_{n}, f\right)
$$

where $t_{n}$ is the time corresponding to the local time spectrum, $f$ is the frequency of the local time spectrum, and $\langle\bullet\rangle$ is the expectation of distribution.

For a nonstationary case, the energy distribution in the time-frequency domain is $S\left(t_{n}, f\right)$ at $t_{n}$ and the entire energy distribution of signal $x(t)$ is constituted as a set $\left\{S\left(t_{1}, f\right), \cdots, S\left(t_{n}, f\right)\right\}$. If $x(t)$ is a nonstationary signal, its time-frequency power spectral density will vary over time. A quantized value $\theta$ is proposed here to represent energy fluctuations in the time-frequency domain. The variance of $\left\{S\left(t_{1}, f\right), \cdots, S\left(t_{\mathrm{n}}, f\right)\right\}$ indicates the level of nonstationarity.

$$
\begin{aligned}
& c_{n}=D\left(S\left(t_{n}, f\right),\left\langle S\left(t_{n}, f\right)\right\rangle_{n}\right) \\
& n=1, \cdots, N, j=1, \cdots, J \\
& \theta=E\left(c_{n}-E(c)\right)
\end{aligned}
$$

where $E[\bullet]$ is the mathematical expectation; $D(\bullet)$ is the calculation method for the distance between the energy distributions, which is defined as follows:

$$
\begin{aligned}
& D(G, H)=(1 \\
& \left.\quad+\int_{\Omega} \log \frac{G(f)}{H(f)} d f\right) \cdot \int_{\Omega}(\widetilde{G}(f)-\widetilde{H}(f)) \log \frac{\widetilde{G}(f)}{\widetilde{H}(f)} d f
\end{aligned}
$$

Equation (12) $\theta$ can be used to describe the deviation of the energy distribution of the measured signal with that of the whole time series using the distance concept in the time-frequency domain, which is consistent with the level of nonstationarity. This definition is widely applicable because it is based on the basic theory of time-frequency analysis.

A flow chart of the entire nonstationarity level assessment process is provided in Figure 1.

\section{Hypersonic Flutter Analysis Framework}

The feasibility of common flutter analysis methods for hypersonic flutter tests is discussed in this section. There are two main structural response analysis methods as applied to flutter tests: structural modal damping and system stability. The former extrapolates the flutter critical velocity from the subcritical response by fitting the dangerous modal damping; 
the latter obtains the flutter boundary by establishing a dynamic model and calculating the stability parameters.

(a) Structural Modal Damping. The concept of modal damping in flutter test analysis is not limited to static structural damping but applies to mixtures of structural damping, aerodynamic damping, material damping, and all the physical quantities which suppress structural vibration. Hypersonic aircraft structural flutter is a kind of self-excited vibration with aerodynamic force as the primary excitation source. This mechanism allows the structural intrinsic modals to change with time over the course of the test. Technically, modal damping is difficult to generalize or estimate reasonably based on the observed vibration response of the structure-especially in the case of multimodal coupling, complex structures, and structural or material nonlinearity. Flutter modal damping is a relatively small value that is highly sensitive to observation noise, calculation error, computer word length, and other effectually uncontrollable factors.

There are a few existing methods for estimating flutter modal damping from structural responses. The half-power bandwidth method, for example, is derived from a singledegree-of-freedom mass spring system that is usually only applicable to single-mode or multimode decoupled systems. Under dense mode or low signal-to-noise ratio (SNR) conditions, the frequency spectrum is irregular, peaks are overlapped, or an abundance of burrs significantly affects the effectiveness of this method. The random decrement technology is also derived from the single-degree-of-freedom mass spring system wherein any response signal can be split into three parts: random response, initial velocity response, and initial displacement response. When the random response and initial velocity response are partially removed, only the initial displacement response is retained as a free decay signal or unit impulse response of the system. Corresponding damping estimates can be obtained via empirical formula or envelope fitting. This method is only fully applicable to systems with single degrees of freedom and requires a set quantity of observed samples. The Miramand method is based on the transfer function theory. When the excitation signal is determinable statistically, all five modal parameters can be obtained through rational function fitting and the calculation of residues. Generally, this method is suitable for both single-mode and multimode situations. Prony methods are based on complex exponential function fitting-there are many deformation algorithms which apply to these methods, for example, the matrix pencil method, which can be used for multimodal signal analysis to directly obtain the frequency and damping parameters.

Traditional damping-based methods mainly work within the frequency domain under the integral transformation concept. The demands for stationarity and length in the data may not be suited to hypersonic flutter analysis. The progression of variable airspeeds in a hypersonic flutter test does not satisfy the assumption of a stationary stochastic process, which is the theoretical basis of these damping methodologies. Some researchers $[27,28]$ have successfully solved modal identification for nonstationary measured signals by using time-domain modeling methods such as ARMA or TVAR, which are mathematically equivalent to the system stability criterion discussed here.

(b) System Stability Criterion. Considering the structure response as the response of a dynamic system, the stability criterion transforms the flutter problem into a system instability problem; i.e., a discrete system which can be used investigates whether the poles of the system transfer function all lie within the unit circle. As opposed to modal damping methods, system stability methods are defined by the coefficients of the eigenpolynomial of the system. The procedure can be decomposed into two steps. The system is first modeled per the structure responses from a flutter test; then the relationships between the flutter criterion and system coefficients are established.

Generally, the ARMA model can be formed by mixing an AR model representing the response series and a moving average (MA) model as the white noise excitation. The difference equation of the ARMA model is

$$
\sum_{m=0}^{p} a_{m} y(n-m)=\sum_{m=0}^{p-1} b_{m} e(n-m)
$$

where $y(n)$ is the structural response signal for the aircraft, $e(n)$ is the noise (described as aerodynamic excitation), and $p$ is the order of the system, which can be calculated from the AIC criterion or based on a priori information such as the actual number of system modals. Based on the measured data, the coefficients $a_{m}(n)$ with $b_{m}(n)$ in (14) are the key factors in evaluating system stability.

There are many system stability criteria with different forms but mathematical equivalence. The most commonly used are Jury's criterion and Lyapunov's criterion. follows:

Jury's criterion defines the square matrix $X_{k}, Y_{k}$ as

$$
\begin{aligned}
X_{k} & =\left|\begin{array}{cccc}
1 & a_{1} & \cdots & a_{k-1} \\
0 & 1 & \cdots & a_{k-2} \\
\cdots & \cdots & \cdots & \cdots \\
0 & 0 & \cdots & 1
\end{array}\right| \\
Y_{k} & =\left|\begin{array}{cccc}
a_{p-k+1} & \cdots & a_{p-1} & a_{p} \\
a_{p-k+2} & \cdots & a_{p} & 0 \\
\cdots & \cdots & \cdots & \cdots \\
a_{p} & \cdots & 0 & 0
\end{array}\right|
\end{aligned}
$$

where the stability of the model system should meet the following conditions:

$$
\begin{aligned}
& F(+1)>0 \\
& F(-1)>0 \\
& F^{+}(k)=\operatorname{det}\left[X_{k}+Y_{k}\right]>0 \\
& F^{-}(k)=\operatorname{det}\left[X_{k}-Y_{k}\right]>0 \quad k=1,2, \cdots p-1
\end{aligned}
$$

Lyapunov's criterion is defined by the matrix $X=\left[X_{i, j}\right]$ :

$$
X_{1, j}=a_{j-1}-a_{p} a_{p+1-j} \quad j=1,2, \cdots, p
$$


When $i=2, \cdots, L$

$$
\begin{aligned}
X_{i, j} & =X_{i-1, j-1}+h_{i j} \\
h_{i j} & =a_{i-1} a_{j-1}-a_{p+1-j} a_{p+1-i} \quad j=i, \cdots, p+1-i
\end{aligned}
$$

The condition of Lyapunov's criterion for system stability is that the matrix $X$ is positive definite, which means

$$
M_{i}>0 \quad i=1,2, \cdots, p
$$

where $M_{i}$ is the principal minor of the matrix $X$.

The system stability criteria above are based on stationary stochastic process theory. To evaluate nonstationary data from hypersonic flutter tests with progressive variable airspeed, and to characterize the time-varying modal parameters for coupling in the test, the coefficients in (14) must also vary with time. Consider the following time-varying parameter model:

$$
y(n)=-\sum_{k=1}^{p} a_{k}(n) y(n-k)+\sum_{k=1}^{q} b_{k}(n) u(n-k)
$$

where the coefficients $a_{k}(n)$ and $b_{k}(n)$ are also the functions of sampling point $n$. Generally, the atmospheric turbulence excitation can be approximated as Gaussian white noise, so (21) can be simplified as a time-varying autoregressive (TVAR) model:

$$
y(n)=-\sum_{k=1}^{p} a_{k}(n) y(n-k)+u(n)
$$

When the dynamic data has been modeled based on the measured signals, the system stability criterion can be calculated by substituting the time-varying coefficients $a_{k}(n)$ into (17) and (20) and then extrapolated to obtain a flutter boundary prediction.

The system stability criteria and time-varying model do not necessitate the assumption of a stationary stochastic process. They are feasible for nonstationary situations caused by hypersonic flutter tests with variable airspeeds. Unlike damping-based methods, however, they lack a clear physical meaning to guide structure design and their accuracy is directly affected by dynamic modeling quality. It is important to select an appropriate modeling algorithm.

(c) Hypersonic Flutter Analysis Framework. According to the characteristics of the hypersonic flutter test and the flutter analysis methods discussed above, we drew a block diagram of the flutter prediction system (Figure 2) which includes nonstationarity level assessment, measured data preprocessing, modal damping estimation, dynamic data modeling, system stability calculation, curve fitting and extrapolation, basic I/O interfacing, and other necessary modules. Preprocessing includes statistical analysis and digital filtering. The modal damping is estimated within a stationary situation to reflect actual engineering requirements. The system stability calculations are designed for the rest of the nonstationary case. The dynamic data modeling module contains several

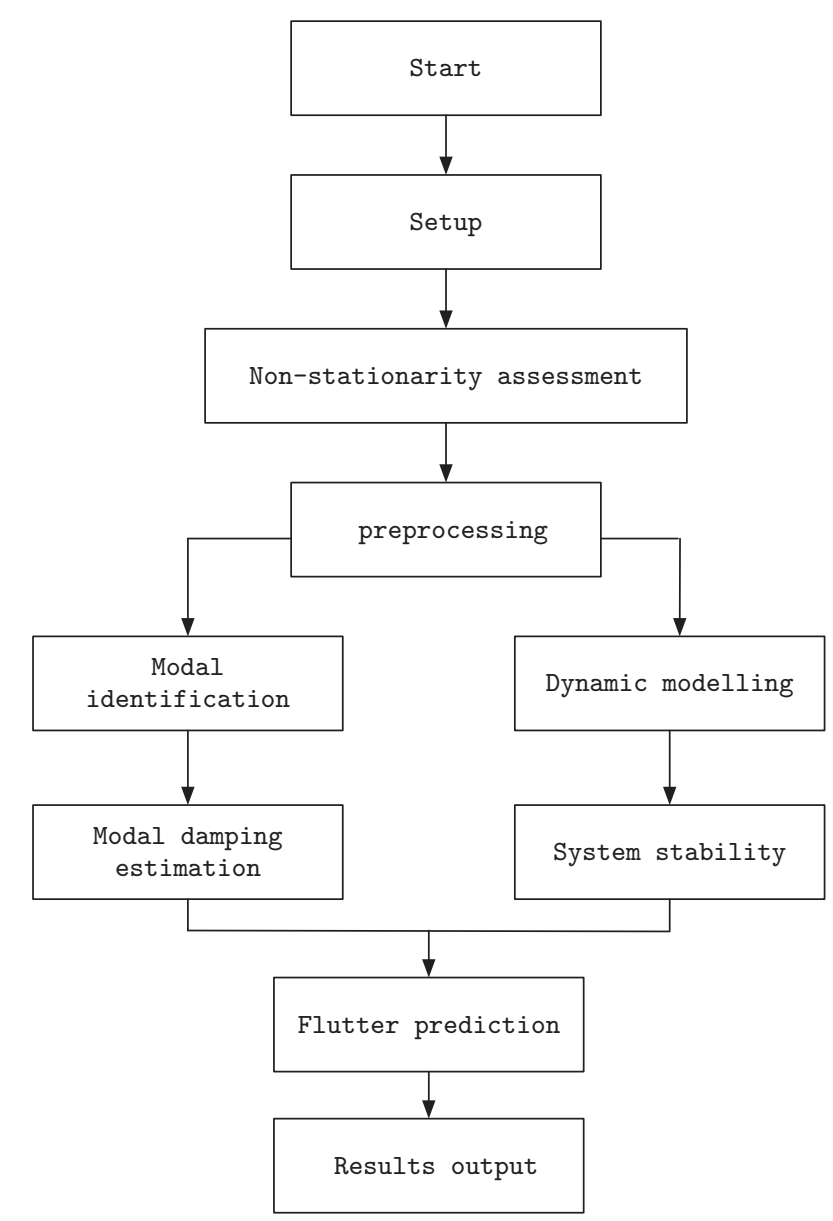

Figure 2: Hypersonic structural frame flutter monitoring and analysis system.

time-varying modeling algorithms based on the adaptive filter theory and Bayes method.

The parameters necessary upon system initiation depend on the characteristics and mechanism of the test aircraft. The nonstationarity of the measured signal is assessed online; then appropriate algorithms are selected accordingly. In the case of nonstationarity, dynamic data modeling is performed after the signal preprocessing; the flutter criteria of the system stability are estimated simultaneously, the confidences of which are according to the level of nonstationarity as determined. Prediction results are then secured by curve fitting and extrapolation with the criteria against the Mach number.

\section{Data Simulation}

(a) Nonstationary Simulation. To validate our nonstationarity level assessment based on the energy distribution in the timefrequency domain, we generated two nonstationary signals with variable amplitude and frequency. The amplitude of signals does not affect the nonstationarity level, so the rate of variation speed of the amplitude simulated was considered to be the independent variable. We investigated the start frequencies, termination frequencies, and rates of speed 


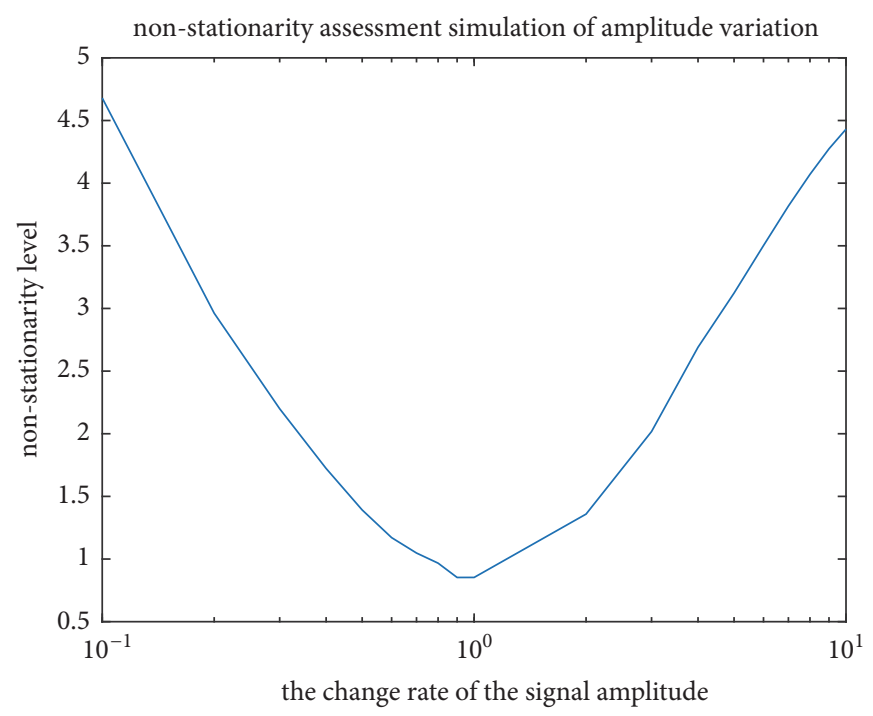

FIgURE 3: Nonstationarity of variable amplitude signal.

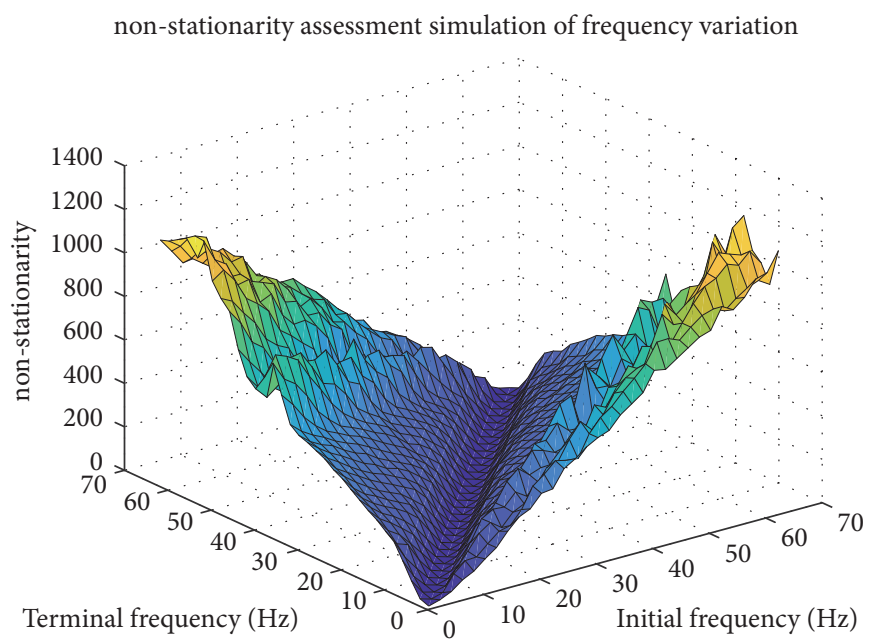

Figure 4: Nonstationarity of variable frequency signals.

variation in a variable frequency case. The results are shown in Figures 3 and 4.

In Figure 3, the abscissa represents the change rate of the signal amplitude from the beginning to the end, while the ordinate represents the nonstationarity as calculated by the proposed method. The middle point of the curve corresponds to the stationary signal, which has the minimum nonstationarity level. The left side of the minimum point corresponds to the signal with amplitude that decreases over time; the right side shows the signal with amplitude that increases over time. The near-linear symmetry on both sides of the curve indicates that the nonstationarity brought about by changes in amplitude and other factors is consistent and has a direct proportion to the change rate.

In Figure 4, the axis $\mathrm{X}$ represents the initial frequency of the signal, the axis $\mathrm{Y}$ is the terminal frequency, and the axis $\mathrm{Z}$ is the nonstationarity as calculated. The minimum value is a line at the bottom of the tridimensional plane of face, where the starting frequency and ending frequency are equal. The nonstationarity gradually rises on both sides of the plane and exhibits strong linearity and symmetry characteristics. To this effect, the proposed assessment method has good indicative significance.

(b) Flutter Test Simulation with Variable Speed. According to the general mechanism of structural flutter, that is, the coupling of two-order structural modes, the structural modes change over time throughout the flutter process. We selected a numerical simulation to simulate the dynamic process of said structural response and the signals of the flutter test with variable speed. $f_{1 t}, f_{2 t}, \rho_{1 t}, \rho_{2 t}$ represent the frequencies and damping factors of the two-degree-of-freedom structural 


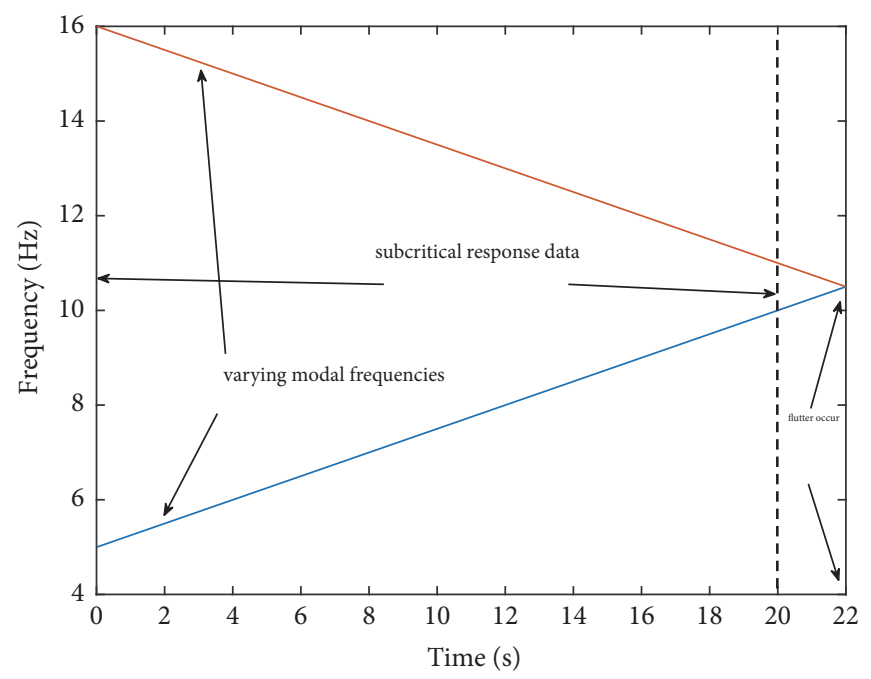

FIGURE 5: Varying modal frequencies in nonstationary case.

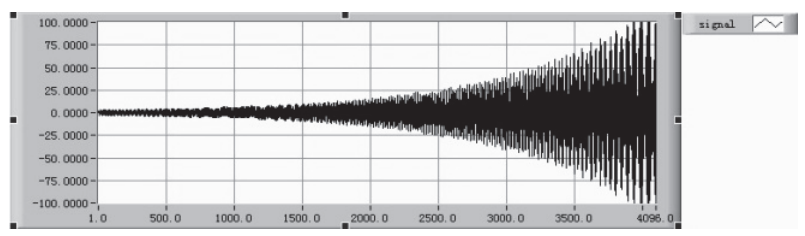

FIGURE 6: Temporal history of simulated signals.

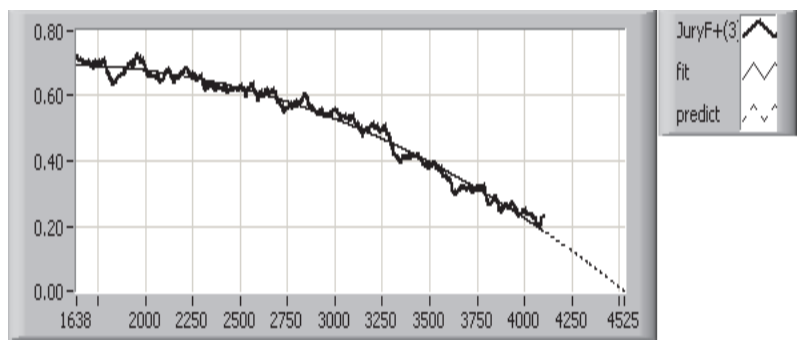

FIGURE 7: Jury's criterion curve and extrapolation.

modes. If the modal frequencies of the dynamic system gradually converge while the modal damping decreases at a certain slope, the dynamic system changes from stable to unstable. For example, consider two frequencies start from $f_{11}=5 \mathrm{~Hz}, f_{21}=16 \mathrm{~Hz}$, converge, and end at $f_{1 n}=9 \mathrm{~Hz}$, $f_{2 n}=12 \mathrm{~Hz}$, where $\mathrm{n}$ is the length of the samples; the modal damping descends from $\rho_{11}=0.100, \rho_{21}=0.05$ to $\rho_{1 n}=0.010, \rho_{2 n}=0.005$. The modal frequencies of the dynamic system converge as shown in Figure 5, while the modal damping decreases at a respective slope. The sample index serves as an indicator of airspeed acceleration due to the linear relation between the sample index and the modal coupling. The corresponding design flutter point is 4,451 . Flutter tests are generally conducted under subcritical conditions, so we selected 4,096 samples in the front as structural response signals under subcritical conditions (Figure 6).

We used the proposed hypersonic flutter monitoring and analysis system to process this signal. Figure 7 shows Jury's criterion with curve fitting and extrapolations as an example. The stability of the dynamic system during flutter is clearly reflected in the trend in the figure.

More prediction results from the different criteria are listed in Table 1 to further demonstrate the accuracy of the proposed framework and the system, which effectively meet the requirements of hypersonic flutter tests (especially the performance of $F^{-}(3)$ ).

\section{Application to Flutter Flight Tests with Variable Speed}

We used data from a wind tunnel test and transonic flight flutter test (as hypersonic flutter test data is relatively scarce) to verify the effectiveness and feasibility of the proposed algorithm.

(a) Wind Tunnel Test with Progressive Variable Speed. Compared to a flutter test with actual aircraft, the FBP result in a wind tunnel test is more closely consistent with the design value. The data can also be verified at lower risk. In our test, the sampling rate was $128 \mathrm{~Hz}$ and the acquisition time was 162 seconds. The airspeed was gradually and smoothly increased until flutter occurred. The torsion signals acquired from the strain gauge are shown in Figure 8 by comparison against the variable airspeed shown in Figure 9. The flutter consistently occurred at about 90 seconds when the airspeed was around $28 \mathrm{~m} / \mathrm{s}$.

We first assessed nonstationarity as shown in Figure 10. Fluctuations in nonstationarity were fairly smooth prior to the flutter but dramatically increased after the vibration diverged.

Next, signals of the front 60 seconds were selected as the subcritical condition for flutter prediction with a maximum speed is $22 \mathrm{~m} / \mathrm{s}$ to further test the proposed framework. The results of system stability criteria $F^{-}$(3) at each time point and the FBP from curve fitting and extrapolation are shown in Figure 11. The FBP result is 27.17, which is not only consistent with the physical experiment, but also 


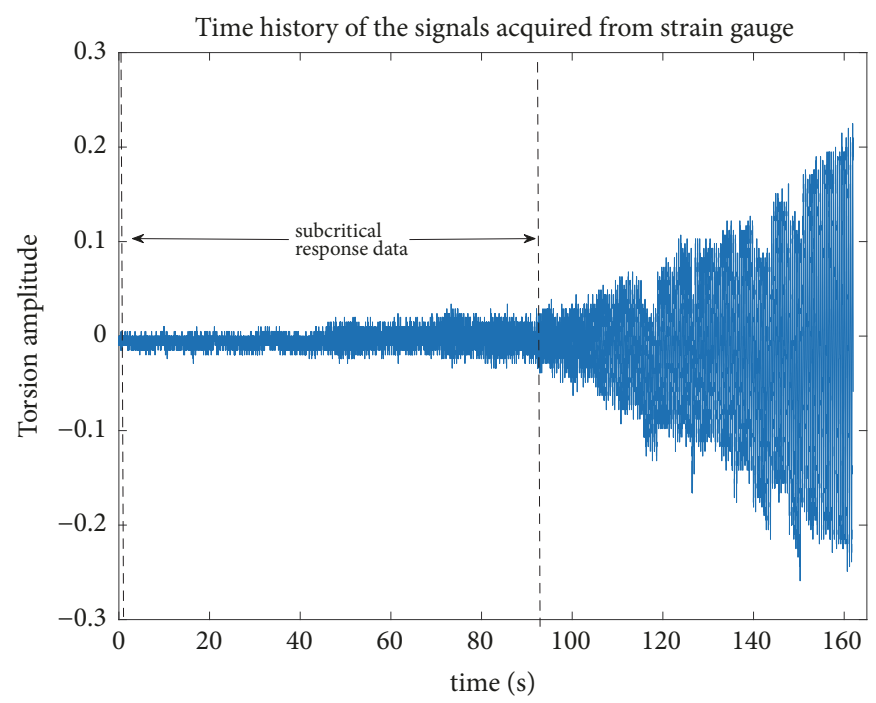

FIGURE 8: Temporal history of torsion signals acquired.

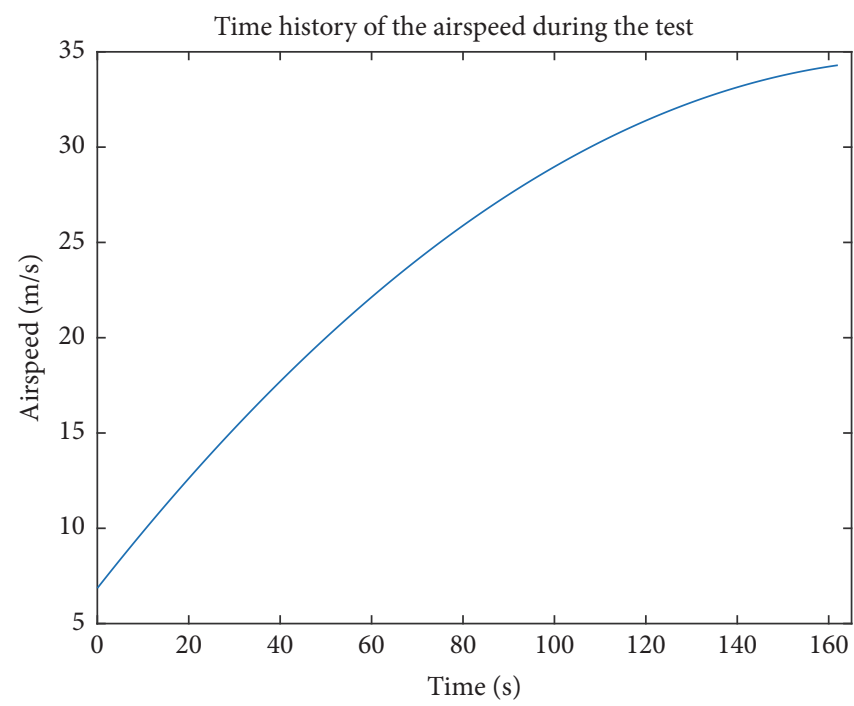

FIGURE 9: Temporal history of variable airspeed during the test.

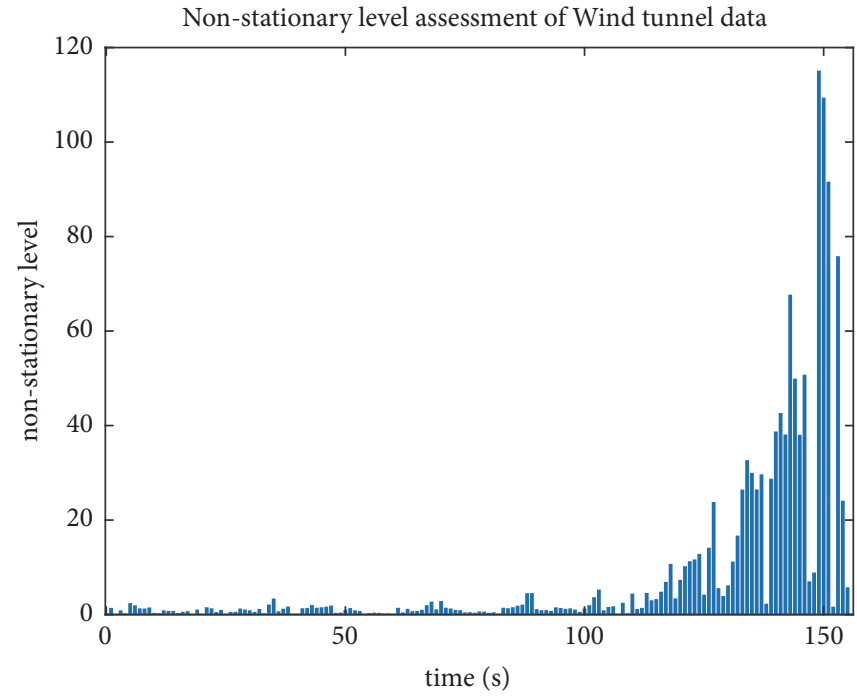

FIGURE 10: Nonstationarity level of signals acquired in variable airspeed flutter test. 


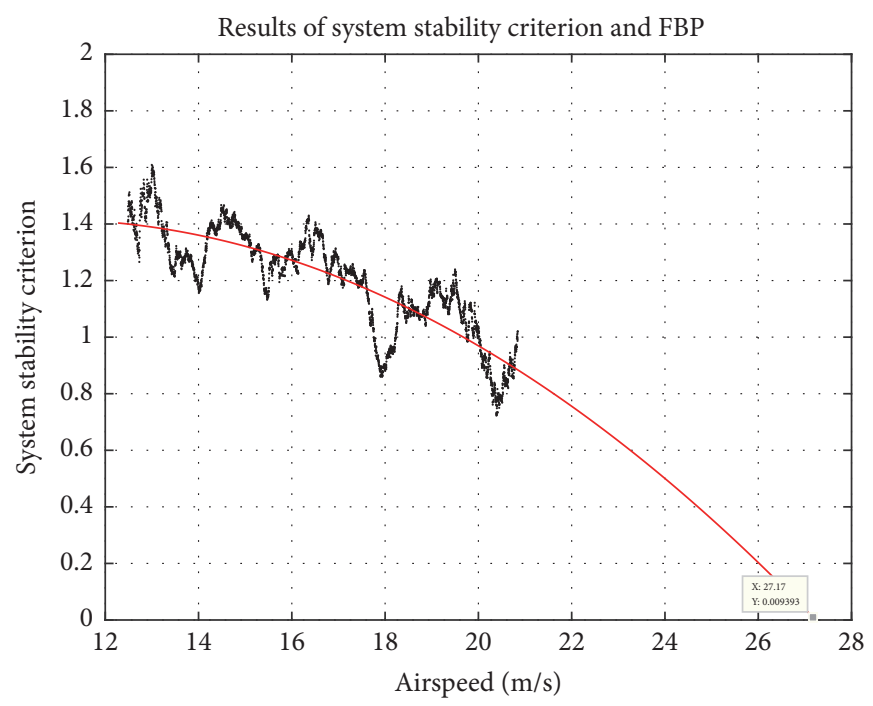

FIGURE 11: System stability criterion and FBP results.

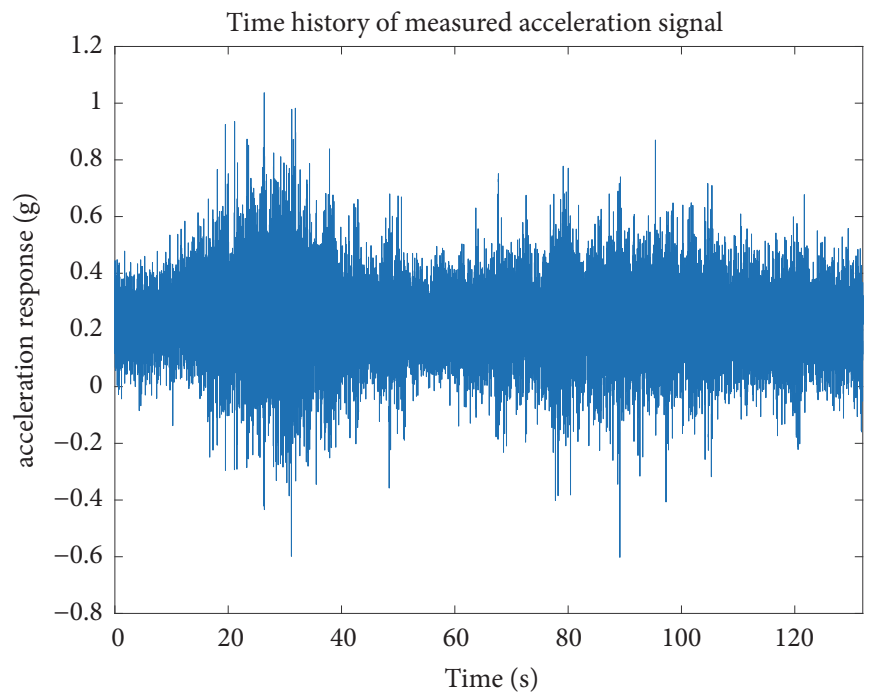

FIGURE 12: Temporal history of measured acceleration signal.

indicative of a monotonous descending tendency at the early stage.

(b) Transonic Flight Flutter Test with Progressive Variable Speed. The selected test data came from a specific type of aircraft transonic flutter flight test which includes both subsonic and transonic periods. Each measured signal was collected from the sensor of an accelerator located on the middle of the left airfoil, as shown in Figure 12, with variable airspeed and flight altitude as shown in Figures 13 and 14, respectively. The sampling rate was $256 \mathrm{~Hz}$ and the acquisition time was 132 seconds.

The nonstationarity levels calculated via the proposed method are shown in Figure 15. The flight speed is far from the flutter velocity, so the signal has lower stationarity than the wind tunnel test signal but is affected by unsteady aerodynamic force or other flight environment characteristics. As shown in Figures 13 and 14, during the 40th second to 60th second time period, the aircraft altimeter and speedometer spiked simultaneously-when the aircraft broke through the sound barrier-accompanied by an abrupt change in nonstationarity level. Above the sound barrier, this effect no longer exists; the nonstationarity level drops back down. This indicates that the proposed assessment method is sensitive to the conditions of measured signals.

We selected orthogonal basis functions method for TVAR modeling in subsequent data processing per the nonstationarity levels as calculated. The system stability criteria at each time point and the FBP results from curve fitting and extrapolation are shown in Figure 16. The maximum airspeed of the flight test must be limited far below the flight envelope, especially for variable airspeed situations, to ensure safety. As opposed to wind tunnel tests, there is no true value for comparison. However, the consistent and monotonous 


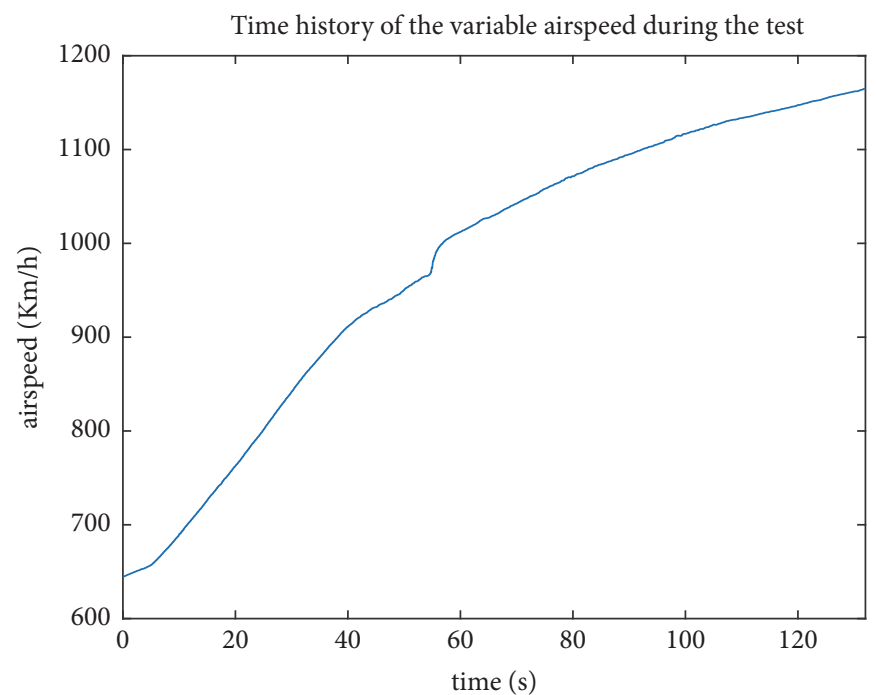

FIGURE 13: Temporal history of variable airspeed during the test (normalized).

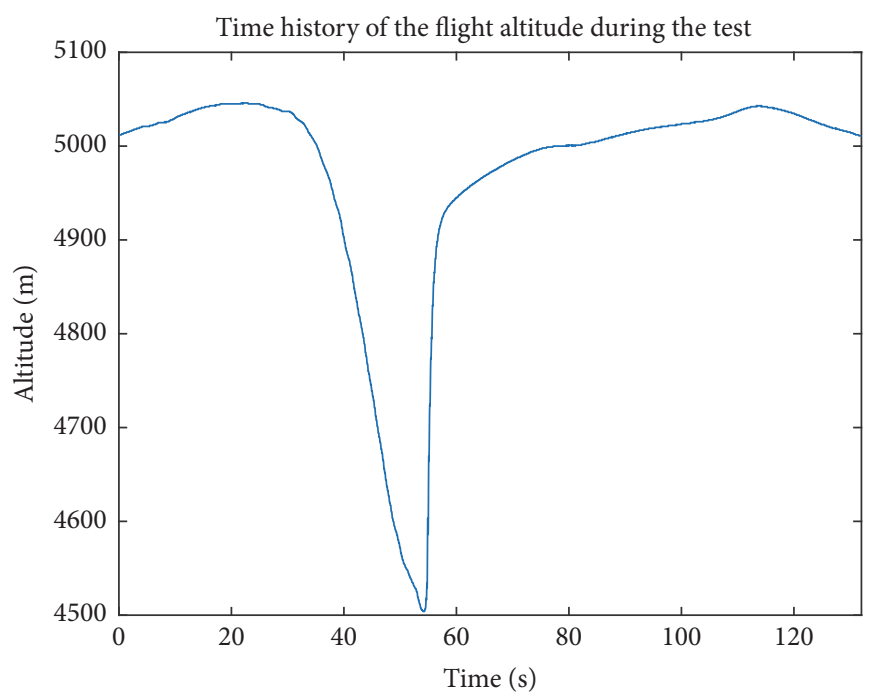

FIGURE 14: Temporal history of flight altitude during the test.

Non-stationary levels assessment of the flight flutter test with variable speed

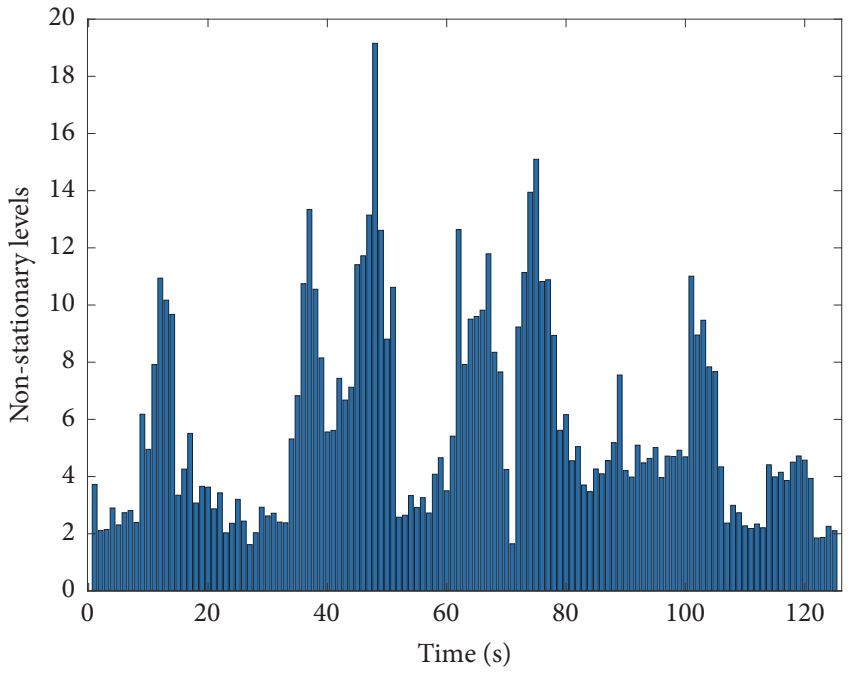

FIGURE 15: Nonstationarity levels of flight flutter test with variable speed. 


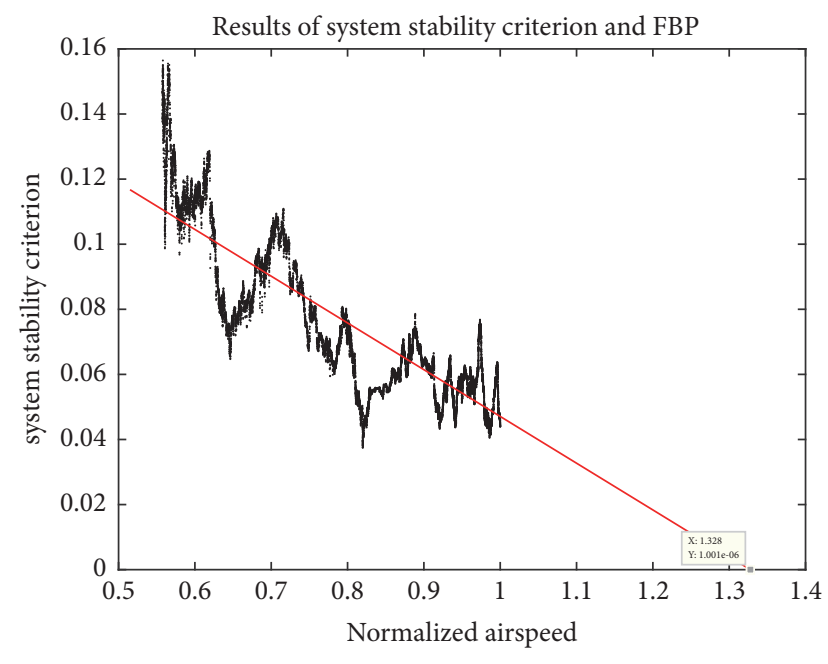

FIGURE 16: Results of system stability criterion and FBP.

descending tendency validates the effectiveness of the proposed framework.

\section{Conclusion}

A flutter analysis methodology based on structural responses of hypersonic flutter tests is proposed in this paper as a response to the inherent drawbacks to existing aerothermoelastic numerical simulations. The fundamental problems with nonstationarity assessment theory and flutter prediction criteria are discussed; then a novel signal processing framework is introduced based on hypersonic engineering. Simulations and experimental results indicate that the proposed assessment method accurately indicates nonstationarity; the proposed flutter analysis framework for hypersonic flutter test also successfully predicts the flutter boundary forming the subcritical turbulence response in a variable airspeed scenario.

\section{Data Availability}

The data used to support the findings of this study are available from the corresponding author upon request.

\section{Conflicts of Interest}

The authors declare that they have no conflicts of interest.

\section{Acknowledgments}

This work is supported by the Aeronautical Science Foundation of China (20151353017), National Natural Science Foundation of China (Grant no. 11302175), and the Fundamental Research Funds for the Central Universities (3102017HQZZ013).

\section{References}

[1] D. Spivey N, High-Temperature Modal Survey of a Hot-Structure Control Surface, NASA TM-215965, 2011.
[2] W. M. Kehoe and H. T. Snyder, Thermoelastic Vibration Test Techniques, NASA TM-101742, 1991.

[3] H. Lee, S. Ma, Y. Chen, Z. Zou, and H. Liu, "Experimental study on compact heat exchanger for hypersonic aero-engine," in Proceedings of the 21st AIAA International Space Planes and Hypersonics Technologies Conference, Hypersonics 2017, China, March 2017.

[4] J. J. McNamara and P. P. Friedmann, "Aeroelastic and aerothermoelastic analysis in hypersonic flow: Past, present, and future," AIAA Journal, vol. 49, no. 6, pp. 1089-1122, 2011.

[5] D. Xie, M. Xu, H. Dai, and T. Chen, "New look at nonlinear aerodynamics in analysis of hypersonic panel flutter," Mathematical Problems in Engineering, vol. 2017, Article ID 6707092, 13 pages, 2017.

[6] T. M. Harris and L. J. Huttsell, "Aeroelasticity research at Wright-Patterson Air Force Base (Wright field) from 1953-1993," Journal of Aircraft, vol. 40, no. 5, pp. 813-819, 2003.

[7] G. H. Morgan, Flutter Tests of Some Simple Models at a Mach Number of 7.2 in Helium Flow, NASA MEMO 4-8-59L, 1959.

[8] V. C. Spain, A Flutter Investigation of All-Moveable. NASP-Like Wings at Hypersonic Speeds, AIAA-93-1315, 1993.

[9] B. R. Cobleigh and M. A. Croom, "Comparison of X-31 flight and ground-based yawing moment asymmetries at high angles of attack," NASA Technical Memorandum, no. 210393, pp. 7-9, 2001.

[10] S. Guo, W. Gao, E. Zhang et al., "Effects of attack angle on starting performance of a hypersonic inlet," in A Methodology for Coupled Thermal Structural Analysis and Structural Design of Scramjet, 2017.

[11] G. M. D. Currao, A. J. Neely, D. R. Buttsworth, and R. Choudhury, "Measurement and simulation of hypersonic fluid-structural interaction on a cantilevered plate in a mach 6 flow," in Proceedings of the 15th Dynamics Specialists Conference, 2016, USA, January 2016.

[12] A. J. Culler and J. J. McNamara, "Impact of fluid-thermalstructural coupling on response prediction of hypersonic skin panels," AIAA Journal, vol. 49, no. 11, pp. 2393-2406, 2011.

[13] L. Huo and T. Yang, "Aeroelastic analysis for response prediction of hypersonic vehicle rudder," in Proceedings of the 21st AIAA International Space Planes and Hypersonics Technologies Conference, Hypersonics 2017, China, March 2017.

[14] H. Torii and Y. Matsuzaki, "Flutter margin evaluation for discrete-time systems," Journal of Aircraft, vol. 38, no. 1, pp. 42-47, 2015.

[15] H. Zheng, J. Zhang, and C. Pei, "A stability criterion method based on neural network and its application on flutter boundary prediction," Engineering Intelligent Systems, vol. 24, no. 3-4, pp. 99-104, 2016.

[16] C.-N. Hsu, "A study on fluid self-excited flutter and forced response of turbomachinery rotor blade," Mathematical Problems in Engineering, vol. 2014, 20 pages, 2014.

[17] A. J. García-Palencia and E. Santini-Bell, "A two-step model updating algorithm for parameter identification of linear elastic damped structures," Computer-Aided Civil and Infrastructure Engineering, vol. 28, no. 7, pp. 509-521, 2013.

[18] Q. Zhu, Y. L. Xu, and X. Xiao, "Multiscale modeling and model updating of a cable-stayed bridge. I: modeling and influence line analysis," Journal of Bridge Engineering, vol. 20, no. 10, 2015.

[19] P. Górski, "Investigation of dynamic characteristics of tall industrial chimney based on GPS measurements using Random Decrement Method," Engineering Structures, vol. 83, pp. 30-49, 2015. 
[20] S. A. Hosseini Kordkheili, S. H. Momeni Massouleh, S. Hajirezayi, and H. Bahai, "Experimental identification of closely spaced modes using NExT-ERA," Journal of Sound and Vibration, vol. 412, pp. 116-129, 2018.

[21] T. Nahom, T. Kogan, M. Iovnovich, and D. E. Raveh, "Evaluation of an ARMA flutter boundary prediction method based on discrete-time structural responses to turbulence excitation in flight-tests," in Proceedings of the 56th Israel Annual Conference on Aerospace Sciences, IACAS 2016, Israel, March 2016.

[22] S. T. Navalkar and J.-W. van Wingerden, "Nuclear normbased recursive subspace identification for wind turbine flutter detection," IEEE Transactions on Control Systems Technology, vol. 26, no. 3, pp. 890-902, 2017.

[23] H. Zheng, J. Liu, and S. Duan, "Flutter test data processing based on improved hilbert-huang transform," Mathematical Problems in Engineering, vol. 2018, 8 pages, 2018.

[24] K. Kim, I. A. Akbar, K. K. Bae, J.-S. Um, C. M. Spooner, and J. H. Reed, "Cyclostationary approaches to signal detection and classification in cognitive radio," in Proceedings of the 2007 2nd IEEE International Symposium on New Frontiers in Dynamic Spectrum Access Networks, pp. 212-215, Ireland, April 2007.

[25] R. R. Sharma and R. B. Pachori, "A new method for nonstationary signal analysis using eigenvalue decomposition of the Hankel matrix and Hilbert transform," in Proceedings of the 4th International Conference on Signal Processing and Integrated Networks, SPIN 2017, pp. 484-488, India, February 2017.

[26] I. A. Kougioumtzoglou and P. D. Spanos, "Nonstationary stochastic response determination of nonlinear systems: A wiener path integral formalism," Journal of Engineering Mechanics, vol. 140, no. 9, 2014.

[27] T. Uhl, M. Petko, G. Karpiel, and A. Klepka, "Real time estimation of modal parameters and their quality assessment," Shock and Vibration, vol. 15, 8 pages, 2008.

[28] A. Klepka and T. Uhl, "Identification of modal parameters of non-stationary systems with the use of wavelet based adaptive filtering," Mechanical Systems and Signal Processing, vol. 47, no. 1-2, pp. 21-34, 2014. 


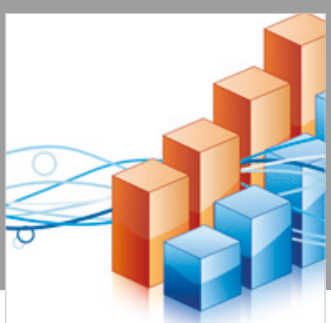

Advances in

Operations Research

\section{-n-m}
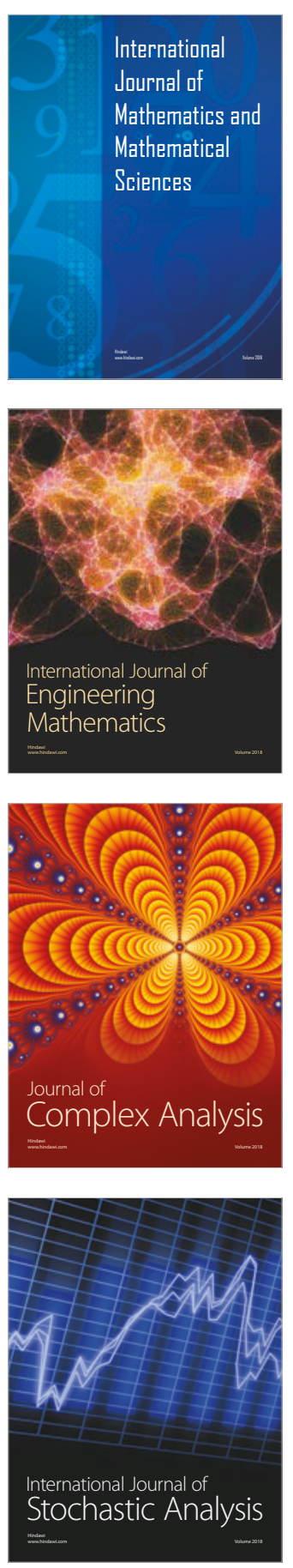
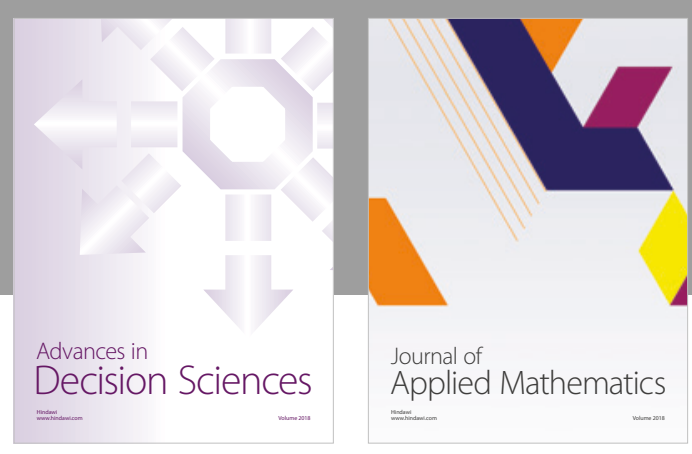

Journal of

Applied Mathematics
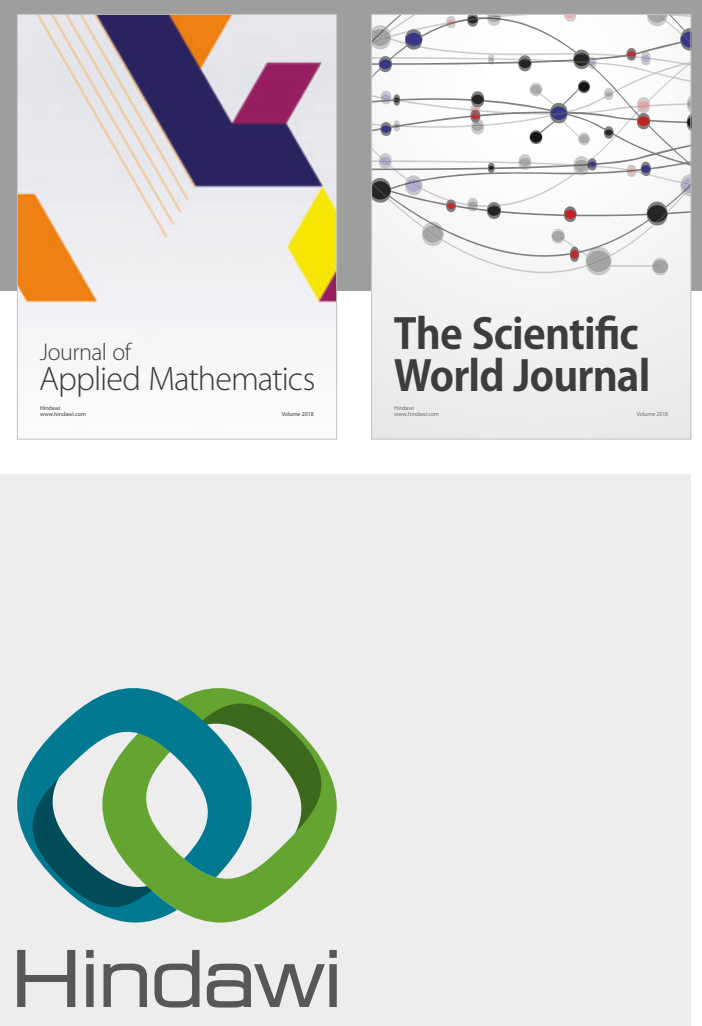

Submit your manuscripts at

www.hindawi.com

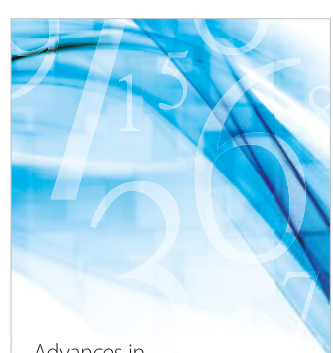

Advances in
Numerical Analysis
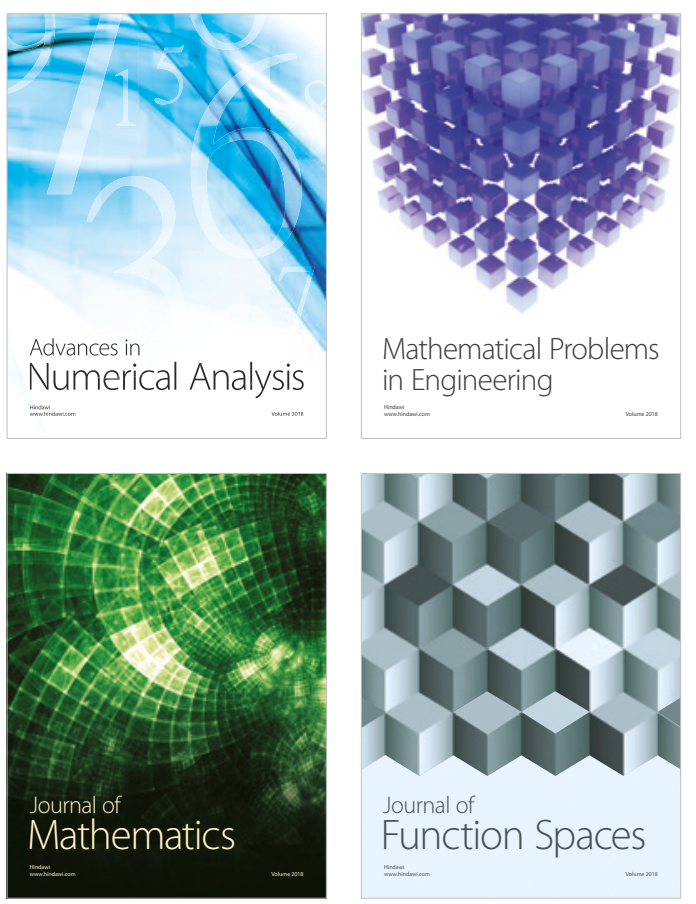

Mathematical Problems in Engineering

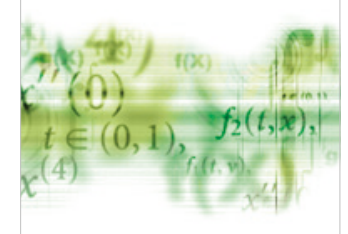

International Journal of

Differential Equations

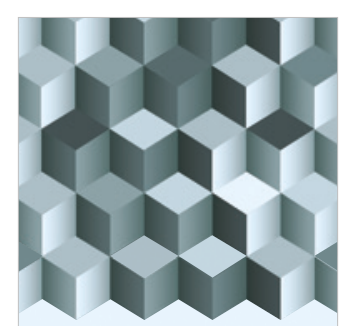

Journal of

Function Spaces

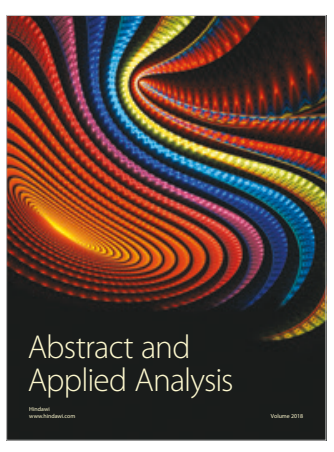

The Scientific

World Journal

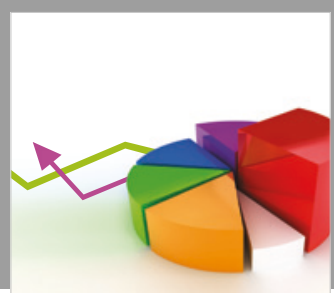

Journal of

Probability and Statistics
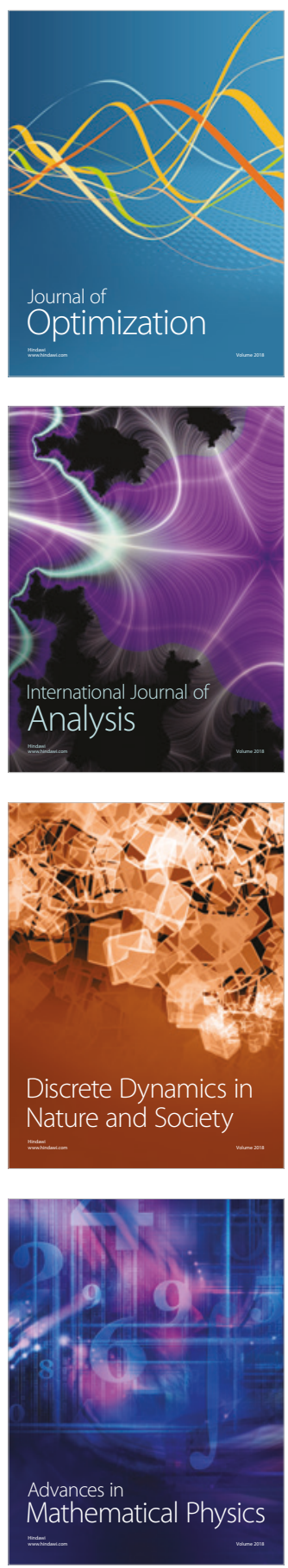